\title{
RUS6, a DUF647-containing protein, is essential for early embryonic development in Arabidopsis thaliana
}

\author{
Nathaniel Perry, Colin D. Leasure, Hongyun Tong, Elias M. Duarte and Zheng-Hui He* (0)
}

\begin{abstract}
Background: The Arabidopsis RUS (ROOT UV-B SENSITIVE) gene family contains six members, each of which encodes a protein containing a DUF647 (domain of unknown function 647) that is commonly found in eukaryotes. Previous studies have demonstrated that RUS1 and RUS2 play critical roles in early seedling development. All six RUS genes are expressed throughout the plant, but little is known about the functional roles of RUS3, RUS4, RUS5 and RUS6.

Results: We used a reverse-genetic approach to identify knockout mutants for RUS3, RUS4, RUS5 and RUS6. Each mutant was confirmed by direct DNA sequencing and genetic segregation analysis. No visible phenotypic differences were observed in rus3, rus4, or rus 5 knockout mutants under standard growth conditions, but rus6 knockout mutants displayed a strong embryo-lethal phenotype. Two independent knockout lines for RUS6 were characterized. The rus 6 mutations could only be maintained through a heterozygote, because rus 6 homozygous mutants did not survive. Closer examinations of homozygous rus6 embryos from rus6/ + parent plants revealed that RUS6 is required for early embryo development. Loss of RUS6 resulted in embryo lethality, specifically at the mid-globular stage. The embryolethality phenotype was complemented by a RUS6::RUS6-GFP transgene, and GFP signal was detected throughout the embryo. Histological analyses with the $\beta$-glucuronidase reporter gene driven by the RUS6 promoter showed tissueand development-specific expression of RUS6, which was highest in floral tissues.
\end{abstract}

Conclusion: Our data revealed that RUS6 is essential for early embryo development in Arabidopsis, and that the RUS gene family functions in multiple stages of plant development.

Keywords: RUS Gene Family, DUF647, Embryo Development, Complementation, Arabidopsis

\section{Background}

A wide variety of internal and external factors regulate and control plant development at various stages [1]. Many basal cellular functions are required for each specific developmental stage. A variety of approaches have been used to identify genes that are required for tissue or organ development, including embryonic, shoot, root, seedling, and flower development. For example, reverse genetics approaches have been successfully used

${ }^{*}$ Correspondence: zhe@sfsu.edu

Department of Biology, San Francisco State University, CA 94132 San Francisco, USA to identify genes essential for embryonic development in Arabidopsis [1, 2]. As of 2020, 510 EMBRYO-DEFECTIVE (EMB) genes, which are required for successful embryo development, have so far been identified and described in Arabidopsis [3]. These genes are required for embryonic viability, with specialized functions ranging from synthesis of macromolecules (DNA, RNA and protein) to cellular structure and metabolism. It is likely that more $E M B$ genes will be identified, and there are an estimated 750 to 1000 EMB genes in Arabidopsis [3-5]. One strategy to uncover additional $E M B$ genes is to focus on gene families where loss of one or more members is known to cause developmental arrest at some stage. 
The ROOT UV-B SENSITIVE 1 (RUS1) gene, which encodes a protein that contains a DUF647 (DOMAIN OF UNKONWN FUNCTION 647), was first identified in Arabidopsis as an essential player in Arabidopsis early seedling development [6]. Knockout mutants for RUS1 displayed an arrested phenotype following germination in a ultraviolet B (UV-B) influenced way [6]. Further studies identified a mutation in a homologous gene, RUS2, which showed identical phenotypes to those of the rus1 knockout mutant [7]. Homozygous rus1 and rus 2 single mutant, and rus1 rus 2 double mutant seedlings displayed an identical post-germination developmental arrest phenotype. This developmental arrest phenotype could be partially rescued by growing seedlings in MS media with high concentrations of vitamin B6, and/or reducing UV-B exposure to MS media plates with standard vitamin B6. Genetic suppressor studies revealed that specific mutations affecting the vitamin B6 binding pocket of ASPARTATE AMINOTRANSFERASE2 (ASP2) suppressed the rus 1 and rus 2 phenotype [8]. These findings suggest that RUS1 and RUS2 may interact with ASP2 to regulate early seedling development through vitamin B6 homeostasis [8].

Interestingly, the RUS2 gene encodes another DUF647containing protein, and both RUS1 and RUS2 share similar expression patterns [7]. Furthermore, RUS1 and RUS2 proteins were shown to physically interact in a DUF647dependent manner, and the interaction appeared to be essential for their physiological function [7]. RUS1 and RUS2 were independently identified as WXR3 (WEAK AUXIN RESPONSE3) and WRX1, respectively, when genetic mutants were screened for defects in auxin response and auxin-related growth defects $[9,10]$. Both wr $x 1$ and wrx 3 mutants accumulated auxin in the hypocotyl and cotyledons, with reduced auxin levels in the root apex $[9,10]$. These studies suggest that RUS1 and RUS2 may play important roles in physiological processes that include UV-B responses, vitamin B6 homeostasis, and polar auxin transport.

About $24 \%$ of all proteins annotated in the Pfam database are categorized as proteins containing a "domain of unknown function" (DUF). Of the 16,295 protein families in the Pfam database, 3,892 are DUF proteins, and the functional roles of these DUF proteins are yet to be experimentally characterized [11]. Taxonomically, DUF-containing proteins are widely distributed in both prokaryotes and eukaryotes. Studies suggested that many DUFs are likely biologically essential [12]. Proteins containing DUF647 (Pfam family PF04884) are widely distributed in eukaryotic species across both the plant and the animal kingdom [7]. Genetic and molecular studies with RUS1 and RUS2 suggested that DUF647 may serve as a protein-protein interacting domain, and the protein-protein interaction between RUS1 and RUS2 via DUF647 is required for Arabidopsis early seedling development [8]. In addition to RUS1 and RUS2, the Arabidopsis genome contains four additional RUS genes named RUS3, RUS4, RUS5 and RUS6, Little is known about the functional roles of these four RUS genes. Assignments of functions for DUF families often depends on making assumptions from the functions of the characterized members. It is currently unknown whether other DUF647-containing proteins are involved in any specific developmental processes. Here we present a comprehensive genetic characterization of the RUS gene family. Our results demonstrate that null mutations for RUS6 result in complete disruption of Arabidopsis embryogenesis by the mid-globular stage. The severe embryonic lethality phenotype in rus6 mutants, and RUS6 expression in flowers, suggest that the RUS gene family plays diverse functional roles in multiple developmental stages from embryonic development to reproduction.

\section{Results \\ The RUS gene family is found throughout eukaryotes and was expanded in algae}

We previously reported that ROOT UV-B SENSITIVE1 (RUS1) and RUS2 are required for post-germination growth in Arabidopsis, and that they likely play a role in vitamin B6 (pyridoxal-5'-phophate) homeostasis. The RUS1 and RUS2 genes both encode proteins that contain a DOMAIN OF UNKNOWN FUNCTION 647 (DUF647) [6]. The Arabidopsis genome encodes for six DUF647-containing proteins (RUS1 through RUS6). RUS proteins are found in most eukaryotic species, including all plants, and most fungus and animals. We previously identified RUS3 as the clear ortholog to the single RUS gene found in most animal genomes [7]. All plant genomes analyzed were found to encode for multiple RUS proteins, usually six or more. Protein sequence analyses identified clear orthologs of RUS1, RUS2, RUS3, and RUS6 in all plant genomes. The genomes of rice and the moss Physcomitrella patens each contained recent duplications of RUS6, but the rice genome lacked a clear RUS4 ortholog, and the P. patens genome lacked a clear RUS5 ortholog. The genome of the gymnosperm Pinus sylvestrus contained orthologs for all six RUS genes (Figure S1). Interestingly, we also identified orthologs for all six RUS genes in the genome of a Charophyte algae, Klebsormidium nitens, which branched from the plant lineage at least 700 million years ago [13]. Therefore, the expansion of the RUS gene family into the current set of six genes occurred long before the evolution of the embryophytes began. 
Identification and analysis of knockout mutants for RUS3, RUS4, and RUS5

In an effort to further understand the functional roles for all RUS members, we screened and identified knockout mutants for RUS3 (AT1G13770), RUS4 (AT2G23470), RUS5 (AT5G01510) and RUS6 (AT5G49820). Potential T-DNA insertional lines were identified in the public database and verified by gene-specific PCR markers. Homozygous knockout mutants were identified for RUS3 (two lines: SALK_135717C and SALK_042033C, which are rus3-1 and rus3-2, respectively), RUS4 (one line: GK447F02-024,530) and RUS5 (one line: SALK_038772C) (Fig. 1). All mutant lines contain T-DNA insertions in exons (Fig. 1) (Figure S2). Homozygous mutants for all three genes (RUS3, RUS4, and RUS5) were isolated, suggesting that mutations in these three genes do not cause embryo lethality (Fig. 1). No noticeable morphological differences were observed between these mutants and the WT (Col-0) plants when grown under standard growth conditions.

\section{Loss of function in RUS6 (AT5G49820) results in embryo lethality}

Two T-DNA insertion lines (GK278G06 and emb1879/ cs16037) were identified for RUS6 (AT5G49820), and verified by PCR markers and direct sequencing (Fig. 2a, b; Figure S2). GK278G06 was obtained from Gabi-Kat [14] (https://www.gabi-kat.de/) and confirmed to have a

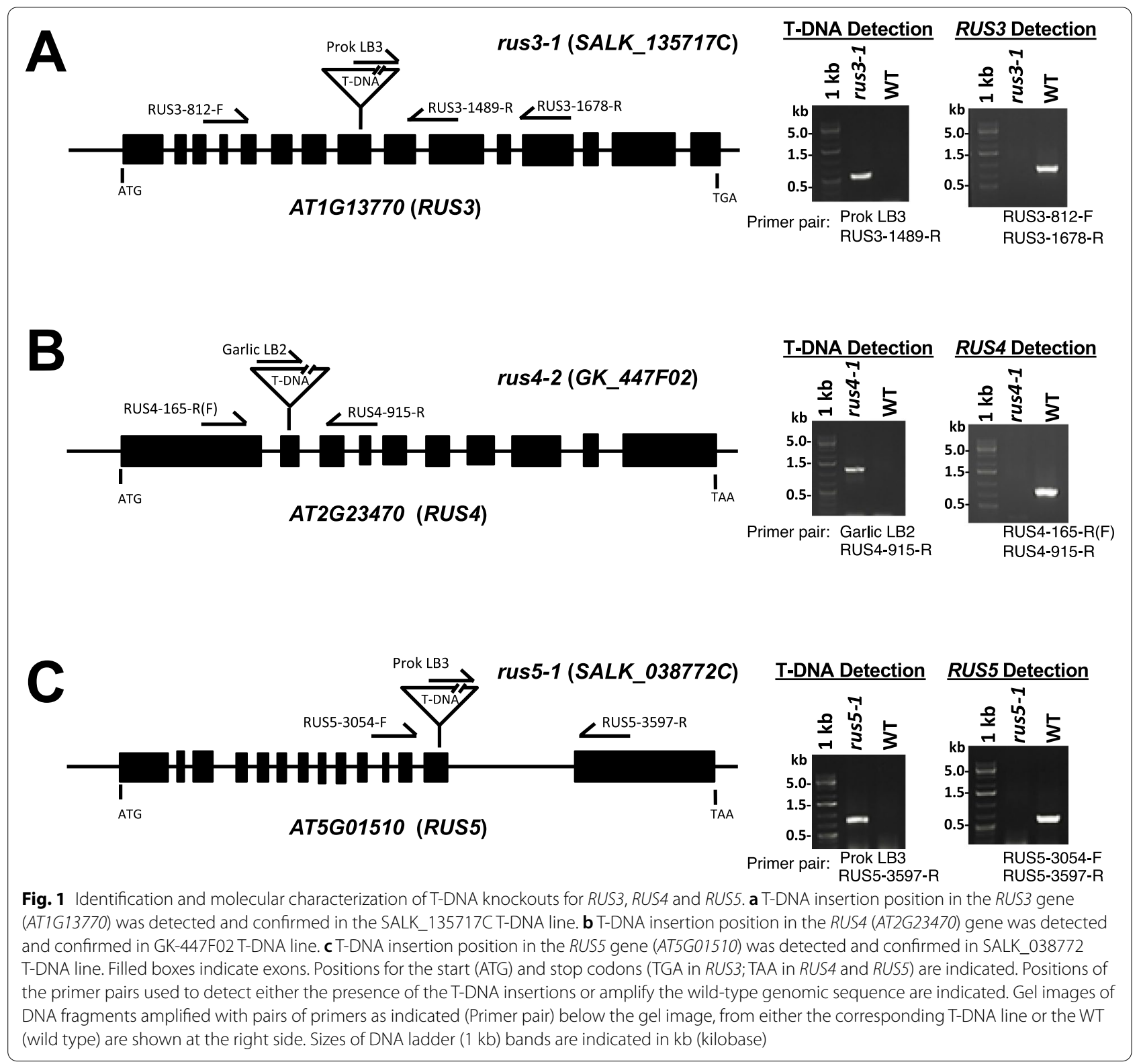




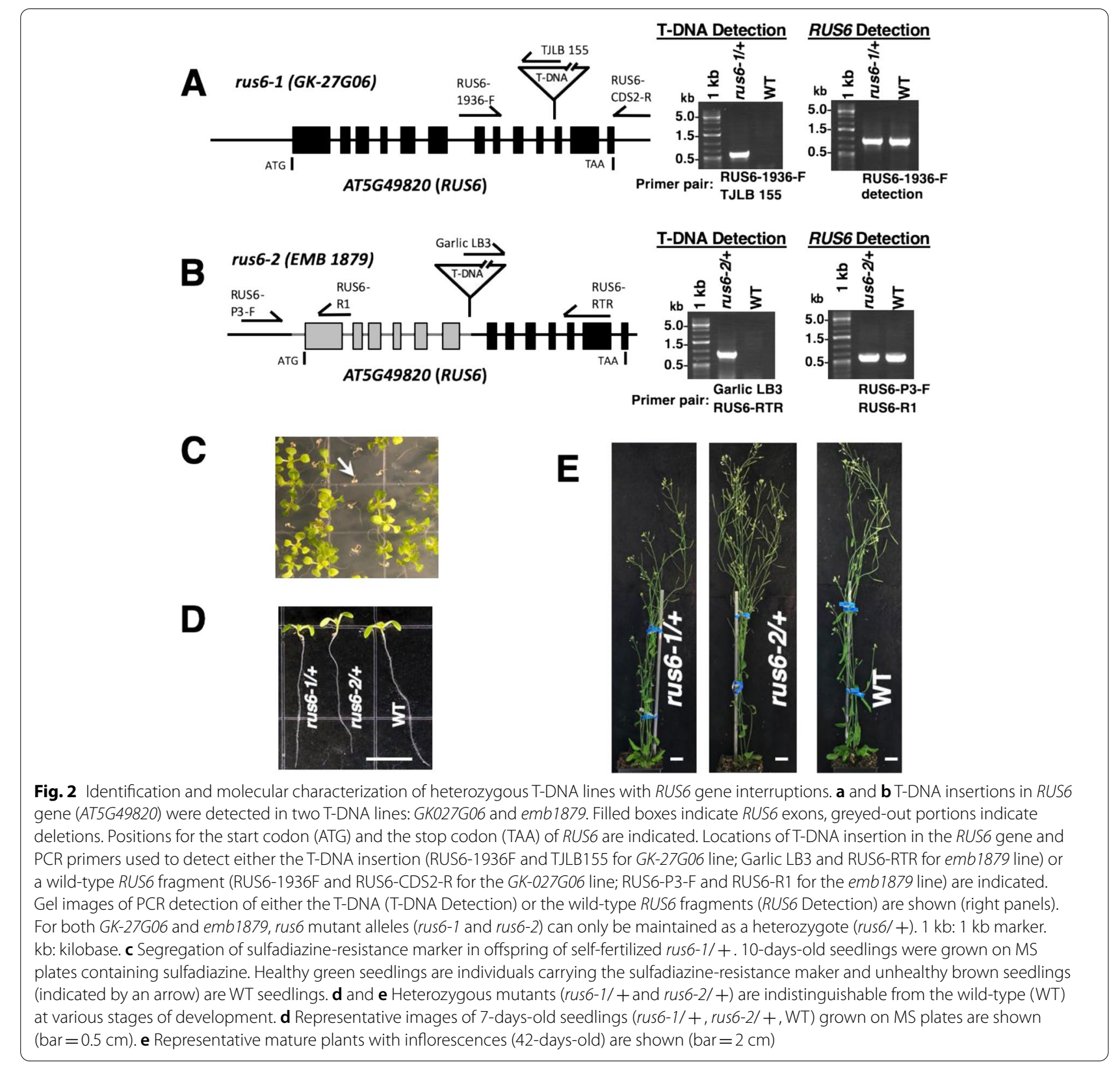

$p A C 106 / p A C 116$ T-DNA insertion in exon 11 (Fig. 2a). The cs16037/EMB1879 line was obtained from ABRC (Arabidopsis Biological Resource Center). The cs16037/ $E M B 1879$ line has a deletion from the promoter region until intron 6, which was replaced by the pCSA104 T-DNA insertion (Fig. 2b). The deletion/insertion was verified by PCR markers and DNA sequencing (Figure S2). After confirming the mutations, we named GK278G06 and cs16037/emb1879 as rus6-1 and rus6-2, respectively (Fig. 2).

We were unable to identify any homozygous rus6 mutants in either of the initial seed stocks for rus6-1 or rus6-2. In order to produce homozygous rus6 plants, rus6-1/ + and rus6-2/ + were each self-fertilized and their progenies were grown. PCR-based genotyping was used to genotype individual progeny, but no homozygous rus6 mutants were identified in the offspring of either heterozygous parent (rus6- $1 /+, n=121$; rus6- $2 /+, n=14$ ), suggesting that homozygous rus 6 mutants are embryo lethal.

The rus6-1 T-DNA insertion contains a sulfadiazine (Sul) resistance gene. We grew the offspring of selffertilized rus6-1/+ plants in the presence of Sul, and observed that $65.27 \%$ of the seedlings displayed Sul resistance and $34.73 \%$ displayed Sul sensitivity $(n=262)$ (Table 1; Fig. 2c). These numbers were consistent with a 
2:1 ratio of rus6-1/+to $+/+$ plants, which is expected if the homozygous rus6-1 plants are absent. A subset $(n=17)$ of the Sul-resistant plants were PCR genotyped and were all identified as rus6-1/+; no homozygous rus6-1 plants were found.

The rus6-2 T-DNA insertion confers Basta (glufosinate) resistance. In agreement with the rus6-1 results, we again observed results consistent with a lack of homozygous rus6-2 plants. $66.20 \%$ of samples displayed basta resistance, and $33.80 \%$ displayed basta lethality $(n=213)$ (Table 1$)$. The basta resistant plants $(n=22)$ that were PCR genotyped were all rus6-2/+ .

The Sul and Basta resistance results conformed to the expected $66.7 \%$ to $33.3 \%$ (2:1) segregation ratio for heterozygous to WT seedlings if homozygous progeny were missing (Table 1). Additionally, seed germination rates were comparable between the mutant lines and wild-type controls, suggesting that the seeds of homozygous rus6 embryos were not produced. Thus, we hypothesized that the lack of rus 6 homozygotes was caused by early embryo lethality leading to seed abortion, rather than failed germination. Taken together, these results suggested that homozygous mutations in rus6 result in embryo lethality.

\section{Loss of function in RUS6 disrupts embryo development, leading to a white developing seed phenotype}

We observed rus6/+mutant plants from germination through maturity, and found that all vegetative parts of the plant were indistinguishable from WT (Fig. 2d, e). To investigate the lack of homozygous rus6 seeds, we opened rus6/ + siliques and characterized the developing seeds inside. While most of the developing seeds were green, similar to wild-type plants, we also observed a high percentage of developing seeds that were white, or brown and wrinkled, depending on the age of the silique (Fig. 3). We suspected that the white developing seeds contained the rus 6 homozygotes, and predicted that they represented $25 \%$ of the seeds in the silique, to fit a 3:1 ratio of green to white seeds [15]. A more extensive phenotypic analysis of developing seeds found 25.59\% white or brown seeds in the siliques of rus6-1/+ plants $(n=895)$ and $23.88 \%$ white or brown seeds in the siliques of rus6-2/+plants $(n=356)$ (Table 2). These results suggested that rus6 homozygotes are embryo lethal, and are the cause of the white developing seed phenotype.

\section{Complementation abolishes the rus6 embryo lethal phenotype}

Our initial analyses of the rus6-1 and rus6-2 mutations strongly suggested that at least one functioning copy of RUS6 is required in Arabidopsis plants. To reduce the possibility of an additional T-DNA insertion somewhere in genome being fully or partially responsible for the rus 6 phenotype, we twice backcrossed rus6-1/+ plants to wild-type Col-0. The rus6 phenotype remained consistent in the purified backcrossed line, which led strong support to the rus6-1 mutation being the cause of the phenotype.

In order to genetically complement the rus6-1 mutation, we created a chimeric $p Z P 222$ construct containing RUS6-GFP driven by the native RUS6 promoter (RUS6::RUS6-GFP). The GFP tag was included for later analysis with fluorescence microscopy. rus6-1/+ plants were transformed using Agrobacterium tumefaciens, and T1 seeds were harvested and plated on antibiotic selection MS media. Two resistant T1 plants were identified and PCR analysis confirmed that they contained the RUS6::RUS6-GFP transgene. T2 seeds were collected from each line, and antibiotic selection and PCR genotyping was performed. We identified rus6-1 homozygous plants in the T2, which contained at least one copy of the RUS6:RUS6-GFP transgene (Fig. 4a, b). The complementation of the rus6 lethality phenotype by RUS6:RUS6-GFP demonstrated that the rus6-1 mutation was responsible for the rus6 mutant phenotype (Fig. 4c).

\section{rus6 homozygous mutations prevent embryo development past the globular phase}

To examine differences in embryo development between the white and green seeds in rus6-1/+ siliques, we performed Differential Interference Contrast microscopy (DIC) on developing seeds. Seeds from the same rus6-1/+ silique were removed, cleared, and examined together, and the results were consistent across siliques analyzed. We initially performed microscopy on seeds from late stage siliques of rus6-1/+ plants, and

Table 1 Segregation of T-DNA insertion in two rus6 knockout lines

\begin{tabular}{|c|c|c|c|c|c|c|c|}
\hline \multirow[t]{2}{*}{ Genotype (line) } & \multirow[t]{2}{*}{ Resistance type } & \multirow{2}{*}{$\begin{array}{l}\text { Number } \\
\text { resistant }\end{array}$} & \multirow{2}{*}{$\begin{array}{l}\text { Number } \\
\text { sensitive }\end{array}$} & \multicolumn{2}{|c|}{ 3:1 Segregation analysis } & \multicolumn{2}{|c|}{ 2:1 Segregation analysis } \\
\hline & & & & $\begin{array}{l}\text { \%resistant / } \\
\text { \%expected }\end{array}$ & $p$ value & $\begin{array}{l}\text { \%resistant / } \\
\text { \%expected }\end{array}$ & $p$ value \\
\hline rus6-1/+ (GK-278G06) & sulfadiazine & 171 & 91 & $\begin{array}{l}65.3 \% / \\
75.0 \%\end{array}$ & $<0.001$ & $\begin{array}{l}65.3 \% / \\
66.7 \%\end{array}$ & 0.63 \\
\hline rus6-2/+emb1879 & basta & 141 & 72 & $\begin{array}{l}66.2 \% / \\
75.0 \%\end{array}$ & 0.003 & $\begin{array}{l}66.2 \% / \\
66.7 \%\end{array}$ & 0.88 \\
\hline
\end{tabular}



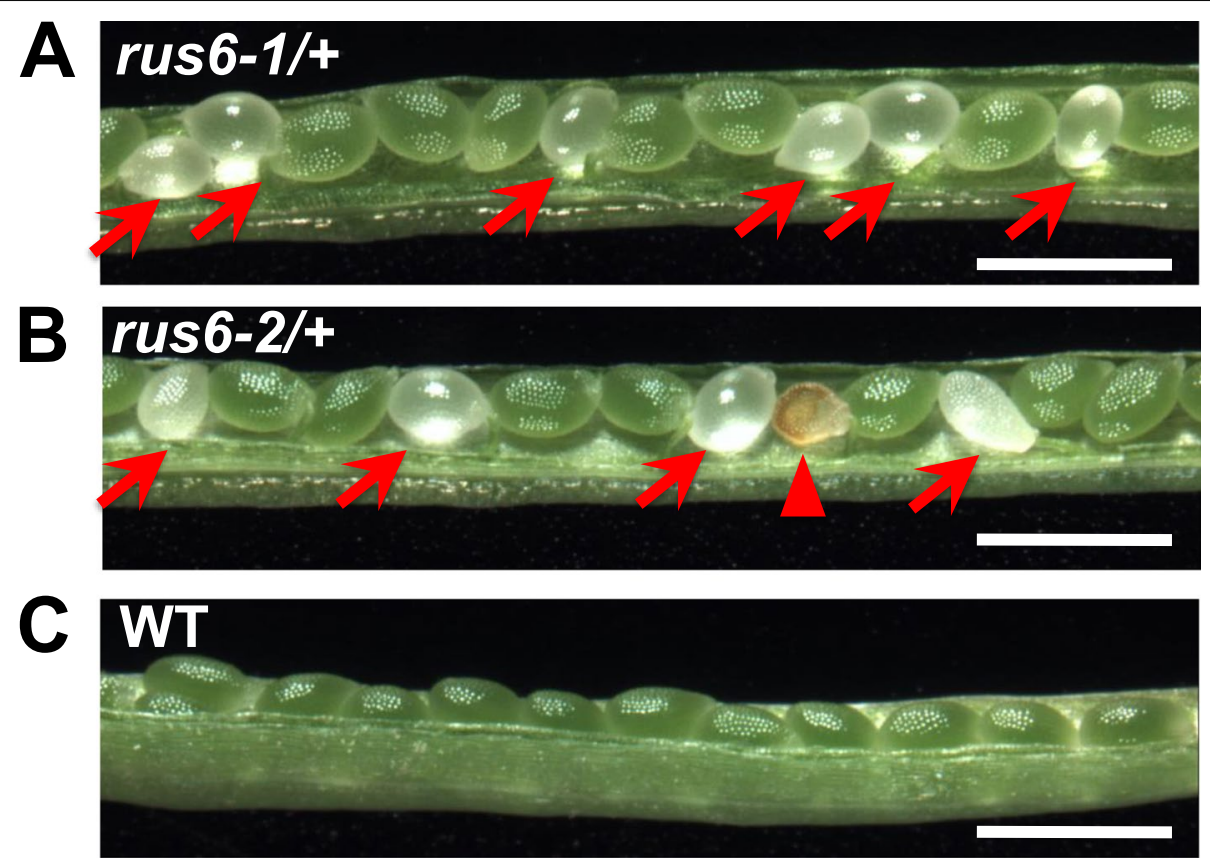

Fig. 3 Embryonic lethality in heterozygous rus6/+ plants. $\mathbf{a}$ and $\mathbf{b}$ Developing siliques with embryos around mid-bent-cotyledon stage to late-bent-cotyledon stage from a rus6-1/ + plant (a) and a rus6-2/ + plant (b) were examined for seed development. c Siliques from WT were used as a control. Normal developing seeds are green and round. White (arrow) and brown (triangle) seeds, indicative of embryo lethality, are present in the siliques of rus6- $1 /+$ and rus6-2/ + plants. Bar $=1 \mathrm{~mm}$

observed that the white seeds completely lacked a detectable embryo. We then examined seeds from siliques of decreasing maturity, which resulted in an increase in the number of white seeds that contained embryos, which were never observed to be past the globular stage. Finally, we observed that in very young siliques all of the white seeds contained globular phase or earlier embryos. These results suggested that the rus 6 embryos were in fact initiated, but degraded and became undetectable after failing to advance past the globular phase.

The rus6 embryos in white seeds were severely delayed, and unable to develop past the globular phase (Fig. 5a, b, c, and d). In contrast, the embryos inside developing green seeds, which were either rus6/ + or wild-type, had normal developmental morphology (Fig. 5e and f). Additionally, the embryos in green seeds in each silique examined were all at a similar stage of development. The rus6 embryos displayed altered morphology, and careful observations determined that they were unable to reach the transition phase. Images shown in $5 \mathrm{~A}, 5 \mathrm{~B}, 5 \mathrm{D}$, and $5 \mathrm{~F}$ were all taken from embryos in the same rus6-1/ + silique. The hypophysis or columella cells were either absent or distorted in such a way as to appear as part of the lower tier (Fig. 5b). Additionally, the suspensors of mutant embryos at this stage were difficult to detect and often appeared to be absent. Figure $5 \mathrm{~d}$ shows an arrested embryo that has not yet begun to deteriorate. Once an embryo starts to deteriorate, it becomes difficult to be imaged for its internal details. Our analyses suggest that rus6 mutant embryo development stalled at the mid- to late-globular phase, and that the embryos subsequently deteriorated leading to failed seed development.

Table 2 Analysis of aborted seeds in the two rus6 T-DNA lines

\begin{tabular}{|c|c|c|c|c|c|}
\hline Genotype (line) & Green (normal) & Brown (aborted) & White (aborted) & $\begin{array}{l}\% \text { of aborted } / \\
\% \text { expected }\end{array}$ & $p$ value \\
\hline rus6-1/+ (GK-278G06) & 666 & 30 & 199 & $\begin{array}{l}25.6 \% / \\
25.0 \%\end{array}$ & 0.69 \\
\hline rus6-2/+EMB 1879 & 271 & 14 & 71 & $\begin{array}{l}23.9 \% / \\
25.0 \%\end{array}$ & 0.62 \\
\hline WT & 460 & 7 & 0 & $\begin{array}{l}1.50 \% / \\
\sim 1\end{array}$ & $<<0.01$ \\
\hline
\end{tabular}



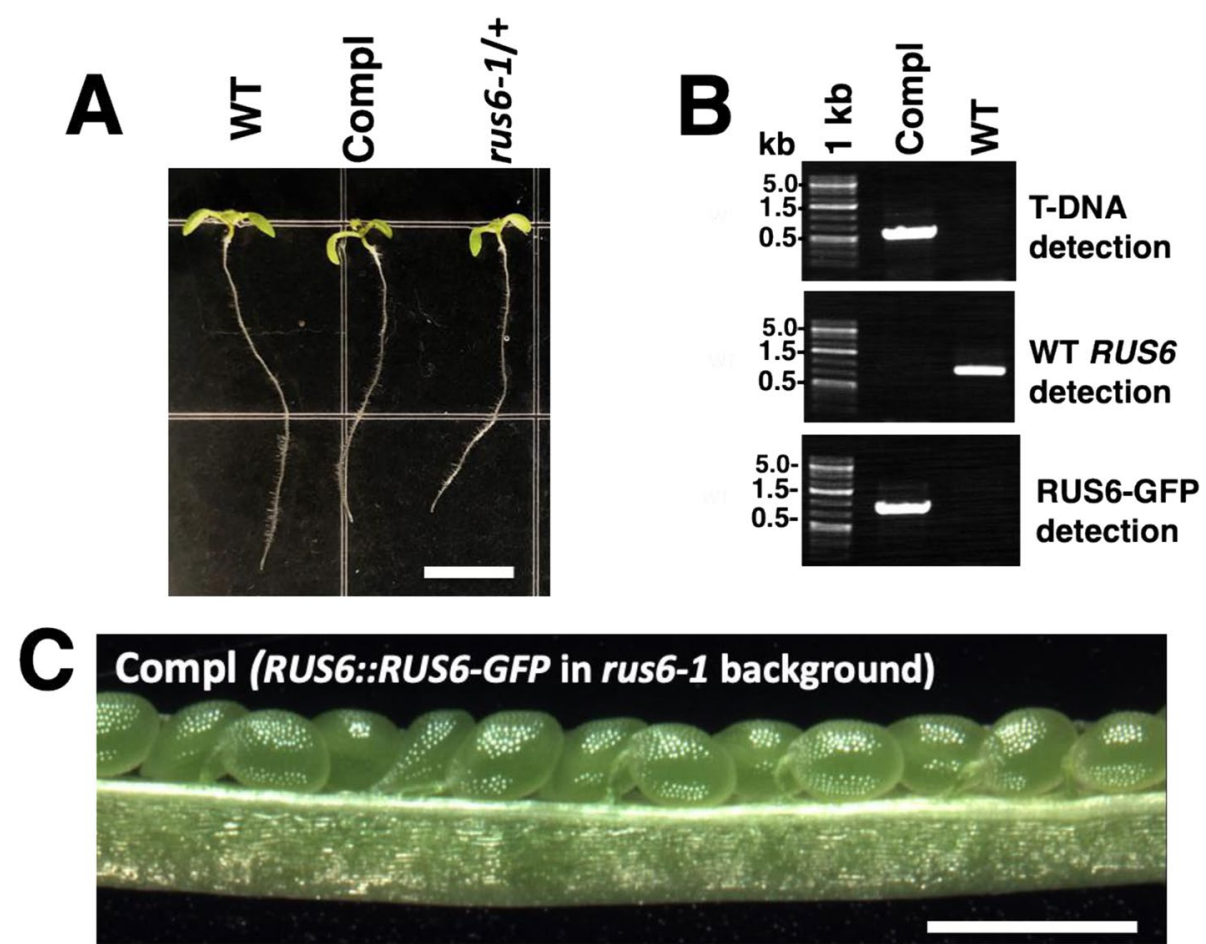

Fig. 4 Complementation of homozygous rus6-1 mutants. rus6-1/ + plants were transformed with a RUS6::RUS6-GFP construct, and homozygous rus6-1 plants carrying RUS6::RUS6-GFP (Compl) were recovered. a Images of 7-days-old seedlings of WT (WT), the complementation line (Compl) (in homozygous rus6-1 background), and the rus6-1/ + line, are shown. Bar $=0.5 \mathrm{~cm}$. b The complementation line carrying RUS6::RUS6-GFP is rus6-1 homozygous. Gel images of specific markers for T-DNA detection (T-DNA detection), WT RUS6 (WT RUS6 detection), and the RUS6::RUS6-GFP transgene (RUS6-GFP detection) are shown. 1 kb: 1 kb DNA ladder. kb: kilobase. c RUS6::RUS6-GFP rescues the rus6-1 white seed phenotype. Image of a representative dissected silique from the RUS6::RUS6-GFP complemented line (in rus6-1 homozygous background) is shown. Bar $=1 \mathrm{~mm}$

\section{RUS6 is expressed in the embryo}

To observe RUS6 expression in vivo, we analyzed GFP fluorescence in homozygous rus6 mutants complemented by our RUS6::RUS6-GFP construct. To minimize the auto-florescence that comes with more developed tissues, we performed laser scanning confocal microscopy on embryos in the late heart to early torpedo stages. We detected GFP fluorescence in complemented embryos that was significantly above the background auto-fluorescence seen in the wild-type control (Fig. 6). Observation at higher magnifications revealed that RUS6 is not specifically localized to the cell wall, nucleus, mitochondria, or any diffuse organelle (Fig. S3). In contrast, fluorescence patterns suggested that RUS6 was mainly localized to some distinct round and punctate structures outside of the vacuole. The TargetP 1.1 predicted that RUS6 localizes to either the chloroplast or other cellular location [16].

\section{RUS6 expression in vegetative and reproductive organs} In order to further evaluate RUS6 expression patterns, we use a $p B I 101$ construct to generate a RUS6::GUS reporter gene. The RUS6 promoter used in this reporter was the same region that was successfully used in the complementation of the rus6-1 mutation. Following Agrobacteria-mediated transformation, selection and PCR analysis confirmed twelve primary (T1) transformants. We performed preliminary GUS staining on all twelve lines, and selected the two with the highest GUS expression levels for further imaging and analyses. T2 plants from line 12 yielded the highest GUS activity in the flowers, while T2 plants from line 1 had the highest expression for all other tissues. RUS6:: GUS expression was observed to be subtle, surprisingly dynamic, and was only detected at specific stages of development.

We stained one- through six-days-old RUS6::GUS lightgrown seedlings grown vertically on M.S. plates. No GUS activity was observed in one day old seedlings, but two days old seedlings showed some degree of GUS activity in the cotyledons (Fig. 7a, b, c). We were unable to detect GUS activity in three- to six-days-old seedlings. Moreover, the GUS activity in two-days-old seedlings was only present in approximately $50 \%$ of the seedlings. This suggests that RUS6 expression was dynamic and temporally specific to a precise stage of development. 

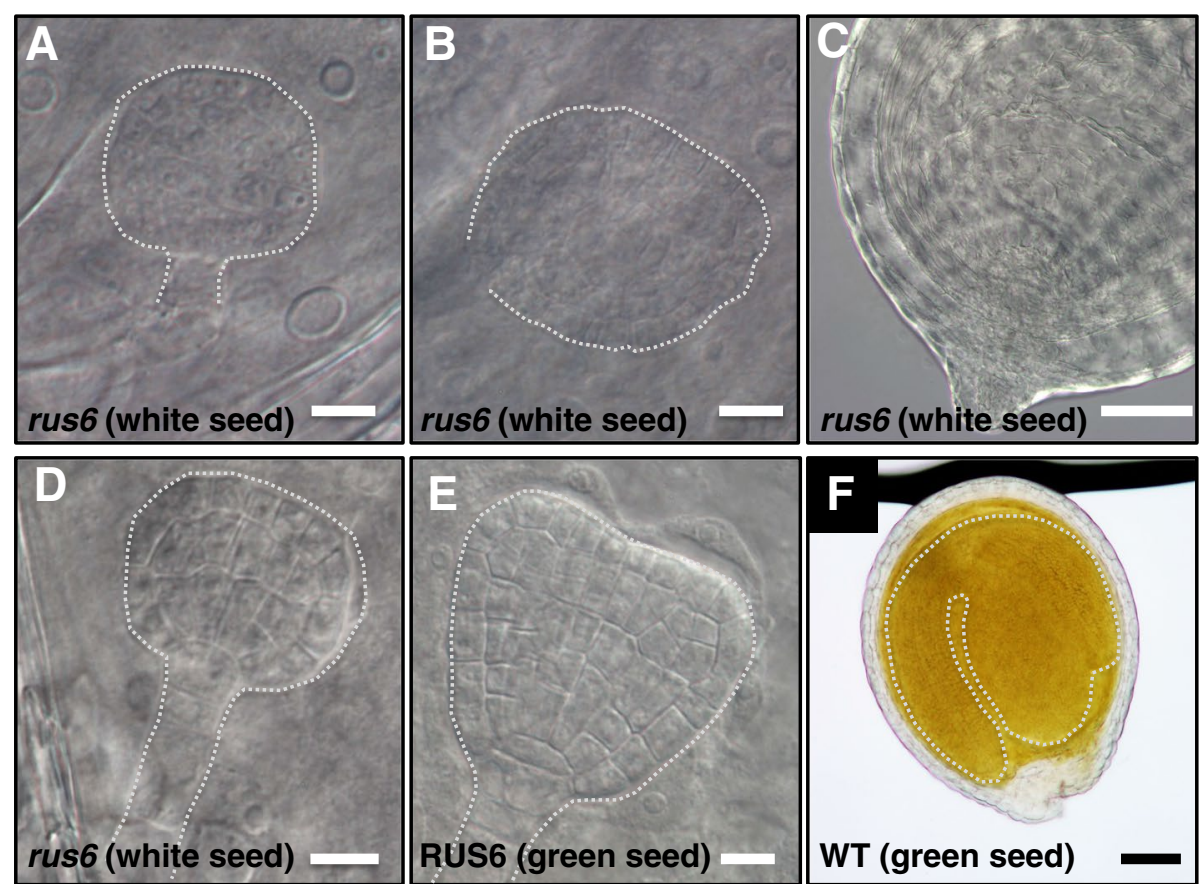

Fig. 5 RUS6 is essential for normal embryonic development. Embryos dissected out from seeds of both rus6-1/+ and WT plants were examined by differential interference contrast (DIC) microscopy. Shapes of the observed embryo are traced with dotted lines to outline the positions of the embryos. Images of embryos from the same rus6-1/ + silique demonstrate that embryos from white seeds are severely developmentally delayed ( $10 \times$ smaller) as compared to those from green seeds, and fail to develop past the mid- to late-globular phase. a-b Defective embryos from white seeds stalled at the mid- to late-globular phase show a distorted/absent suspensor, a lack of identifiable columella, and initial stages of deterioration. c A white seed from a more mature rus6-1/+ silique has no detectable embryo. $\mathbf{d}$ An arrested embryo from a white seed in mid-globular phase that has not yet begun to deteriorate. e A representative transition to early heart stage embryo from a WT silique. No rus6-1/ + white seed embryos were observed at this phase. $\mathbf{f}$ A representative late-bent-cotyledon stage embryo from a green seed shows normal development as compared to white seeds in the same rus6-1/+ silique shown in $\mathbf{a}, \mathbf{b}$, and $\mathbf{d}$. Bar $=5$ microns ( $\mathbf{a}, \mathbf{b}, \mathbf{d}$, and e). Bar $=50$ microns (c and $\mathbf{f}$

In 20-days-old seedlings, GUS activity became clearly defined to the edges of the developing root primordia (Fig. 7e). GUS activity was also observed at this time in some lateral roots (Fig. 7d, e), and very faintly at midlength in the primary root. Interestingly, some lateral roots, root tips, and root junctions showed GUS activity, while others did not. GUS expression did not appear to be based on the length of the lateral root, or any other observable marker of development.

GUS activity was not detected in leaves at any stage of development. However, flowers had the highest detected GUS activity in the plant, which was especially high in the anther (Fig. 7f, g, h, i). GUS activity was uniformly highest in the flower at stage 11, (as defined by Smyth et al., [17]). However, some flowers at later stages showed GUS activity, while others at the same stage of development did not. This pattern persisted even in flowers attached to the same inflorescence stem. Further investigation of dissected anthers revealed that GUS activity was especially high in the tapetum (Fig. 7i).

\section{Discussion}

We have systematically identified and characterized rus3, rus4, rus5, and rus6 knock-out mutants in Arabidopsis. Our study uncovered an essential role of RUS6, which encodes a DUF647-containing protein, in Arabidopsis embryo development. The Arabidopsis genome contains six genes that encode DUF647-containing proteins [7]. Two of the six genes, RUS1 and RUS2, were previously characterized and are known to play critical roles in early seedling development. RUS1 and RUS2 work as functional partners to ensure the heterotrophic Arabidopsis embryo develops into an autotrophic seedling. Both RUS1 and RUS2 were also independently identified in a weak-auxin response genetic screen, suggesting that RUS1 and RUS2 regulate plant development by directly or indirectly affecting auxin distributions $[9,10]$. Recently, a knockdown study of RUS4 via artificial microRNA (amiR) suggested that RUS4 plays a role in Arabidopsis reproductive development [18]. While homozygous rus4 knockout mutants showed no obvious phenotype in their study and ours, down-regulation 


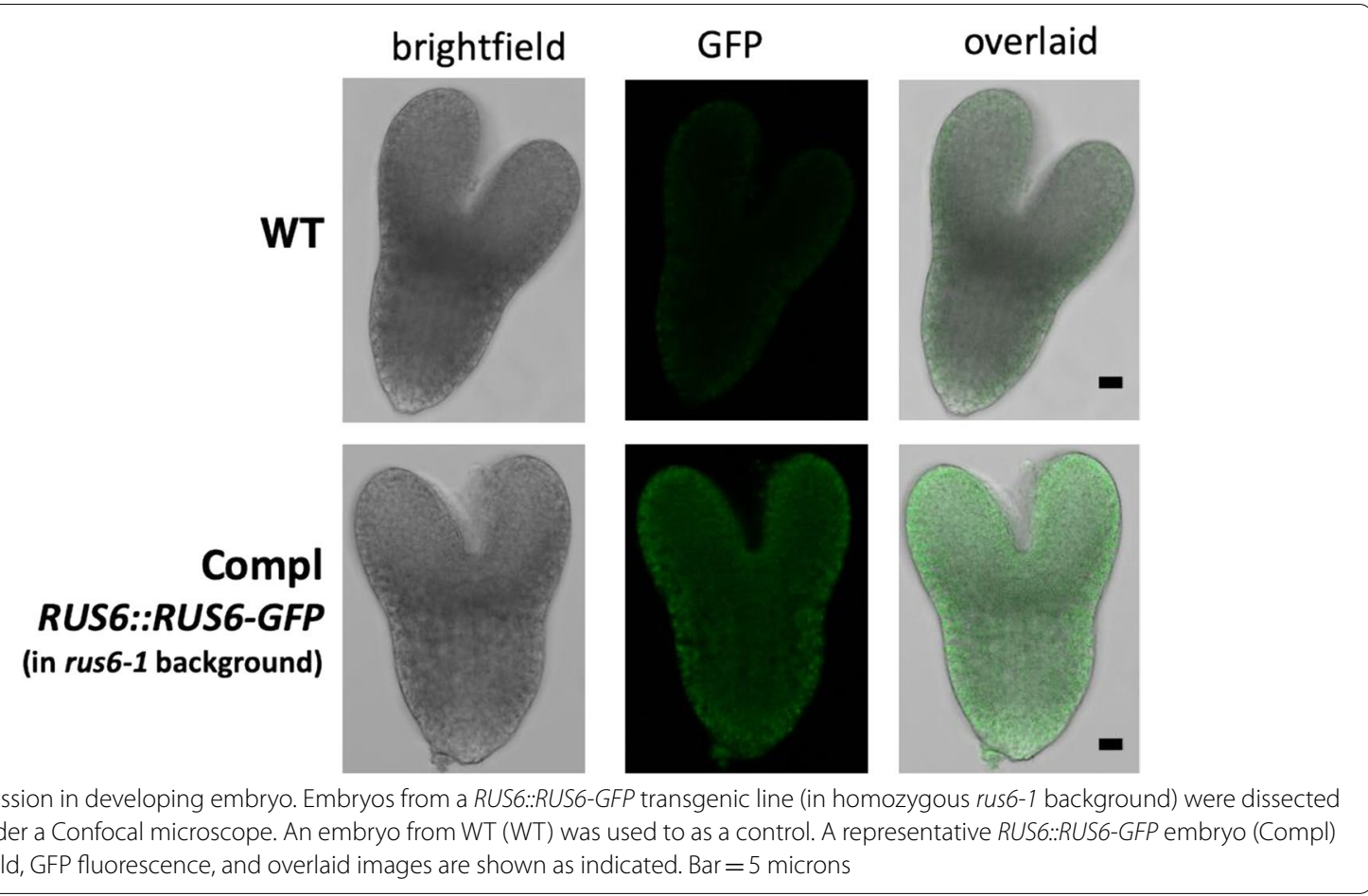

of RUS4 mRNA by the amiR approach disrupted anther dehiscence, likely through the down-regulation of genes such as NST1 and NST2, which are known to play roles in secondary cell wall thickening in the Arabidopsis anther endothecium [18]. In this study, our detailed genetic and molecular characterization of two knockout mutants for RUS6, rus6-1 and rus6-2, clearly identified an indispensable role of RUS6 in Arabidopsis embryonic development.

Embryonic lethality in rus6 knockout mutants was confirmed by several lines of experimental evidence in our study. The rus6-2 allele was identified earlier as an emb (embryo-defective) line with the name of EMB1879 [2], and was tagged as emb by Syngenta (line CS16037, which contains a T-DNA insertion in the promoter region). However, the precise effect of this T-DNA insertion on the gene AT5G49820 (RUS6) was not clear. A further report of embryo-lethality was later removed from the Seed Genes database (seedgenes.org), and recently emb1879/AT5G49820 was excluded from a comprehensive review providing an updated dataset of $510 E M B$ genes [3]. Our analysis in this study provided convincing evidence that emb1979 is in fact an embryo lethal, and should be included in $E M B$ databases. We characterized the emb1879/rus6-2 line along with another available T-DNA line (rus6-1), GK-27G06. No homozygotes could be obtained from either line, and defective seed development was observed in the progeny of heterozygotes for either mutation. These phenotypes are indicative of embryo lethality [19]. In a segregating population, the rus6-1 allele and the rus6-2 allele can be maintained only in individuals that are heterozygous for the mutant allele. Since each allele carries a T-DNA insertion with a specific selectable marker, the presence of the insertion was analyzed on growth media containing the selection reagent, either sulfadiazine or basta, respectively. For both alleles, the segregating populations showed that two thirds of the progeny were heterozygous for either the rus6-1 or the rus6-2 allele, and the remaining one third of the progeny were WT (Table 1). The quantitative data consistently suggested that homozygous rus6 knockout mutants are embryo lethal.

Our study directly detected the nature of the rus6 embryonic lethality through DIC microscopy. While a normal and developed embryo is green, an abnormal and aborted seed is white or brown [20]. Our quantitative analyses of the embryos in the developing siliques of plants heterozygous for either rus6-1 or rus6-2 confirmed that about $25 \%$ of the embryos were white or brown (Table 2). The ratios of the aborted embryos to the normal embryos further support the conclusion that rus6 knockout results in embryonic lethality. In addition, further DIC microscopic examinations of the dissected white seeds demonstrated that embryo development appeared to arrest at stages prior to the transition stage. The aborted embryos showed various abnormalities, including distorted suspensors, or a lack of columella and/or suspensor. In some cases, no visible embryos were observed. It is highly likely that RUS6 

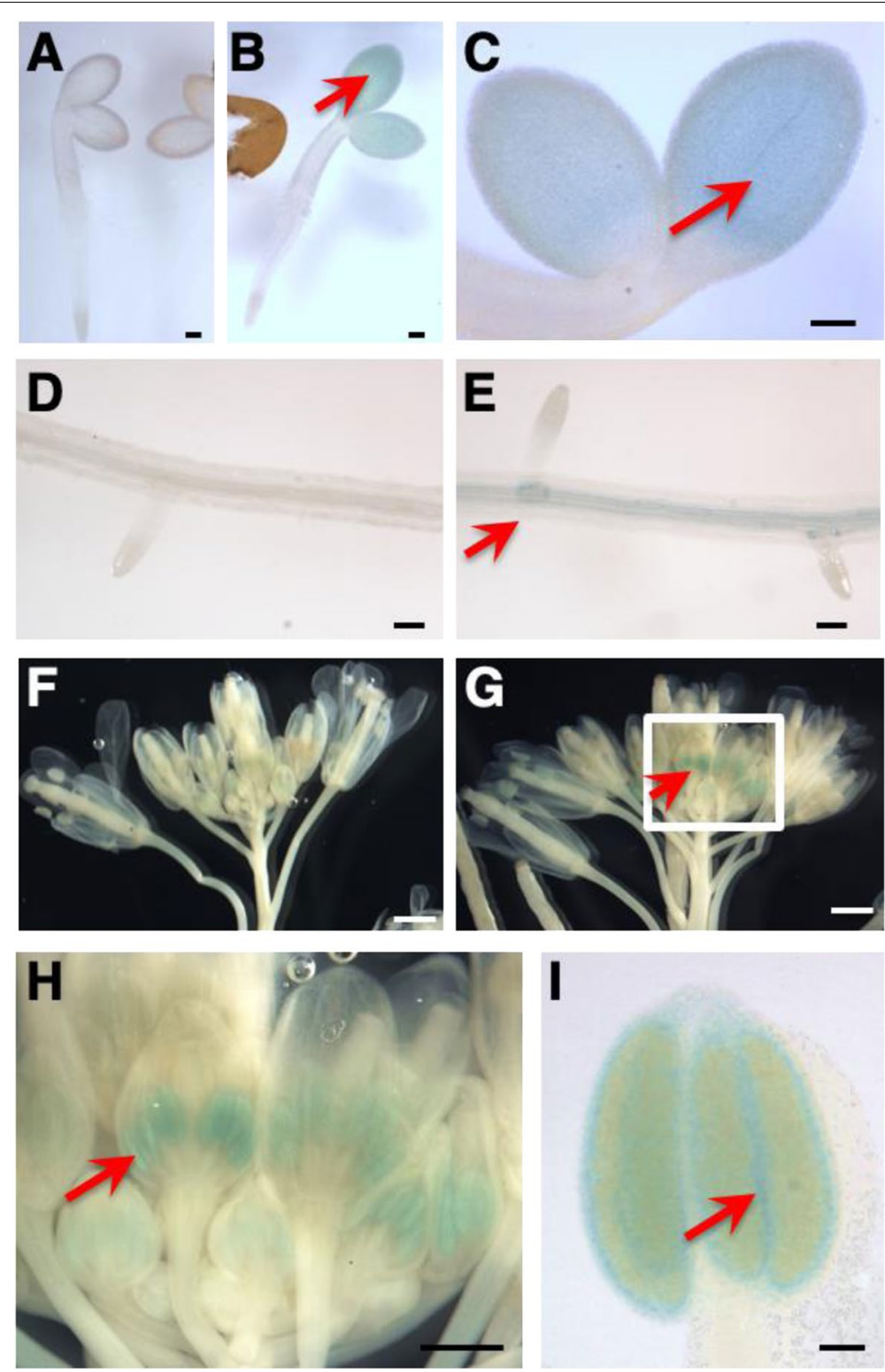

Fig. 7 Organ- and tissue-specific expression of RUS6 expression. $\mathbf{b}, \mathbf{c}, \mathbf{e}, \mathbf{g}, \mathbf{h}$ and $\mathbf{i}$, Images of histochemical staining of RUS6::GUS transgenic plants at various stages of development are shown. Plant organs or whole seedlings were incubated with 5-bromo-4-chloro-3-indolyl beta-D-glucuronide (X-Gluc) to detect $\beta$-glucuronidase activity (GUS), and then cleared before imaged. Locations of GUS staining are indicated by arrows. a WT seedlings are shown $(\mathrm{bar}=0.1 \mathrm{~mm})$. $\mathbf{b}$ and $\mathbf{c}$ GUS staining in 2-days-old transgenic seedlings $(\mathrm{bar}=0.1 \mathrm{~mm})$. $\mathbf{d}$ GUS staining of WT roots as a negative control (bar $=0.1 \mathrm{~mm}$ ). e GUS expression in roots (20-days-old seedling) (bar $=0.1 \mathrm{~mm})$. $\mathbf{f W T}$ flower negative control (bar $=1 \mathrm{~mm})$. $\mathbf{g}$ GUS expression in flowers (bar $=1 \mathrm{~mm}) \cdot \mathbf{h}$ Inset of the boxed area in $(\mathbf{g})(\mathrm{bar}=0.5 \mathrm{~mm}) . \mathbf{i}$ GUS staining in anther $(\mathrm{bar}=0.5 \mathrm{~mm})$

functions at the early stages of embryonic development. As the globular stage was the latest stage detected in the rus6 embryos, RUS6 appears to be required for the embryo to pass through to the transition stage. RUS6 is known to be expressed in various tissues and at various developmental stages including embryo development $[21,22]$. Proteomic analysis suggested that the RUS6 protein (Uniprot \# Q93YU2) is ubiquitously expressed in all tissues analyzed (https://www.proteomicsdb.org/ proteomicsdb/), but the highest RUS6 protein expression was found in mature embryos and pollen [21, 22]. Our RUS6-GFP Confocal data further demonstrated that RUS6 is ubiquitously expressed in late-torpedo stage embryo (Fig. S3). Although high levels of RUS6 expressions are found in pollen, no phenotypes related to gametophyte development were observed in rus6 knockout mutants. Our genetic data suggested that rus6 gametophytes appeared to be functional and could complete 
fertilization at a normal rate. However, the embryos that were homozygous for either rus6-1 or rus6-2 failed to develop beyond the globular stage to form seeds. RUS6 transcript level is also abundant during seed germination and early seedling development [23]. Whether and how RUS6 functions in early seedling development is yet to be analyzed. We complemented the homozygous rus 6 mutant with a construct containing the RUS6 native promoter (RUS6::RUS6-GFP). To further understand the role of RUS6 in other developmental stages, a transgenic line in the homozygous rus6 background carrying RUS6-GFP driven by an inducible promoter can be created in the future.

Although we have clearly established the requirement of RUS6 in Arabidopsis early embryo development, how RUS6 functions during this stage is currently unclear. A number of genes which have been documented as $e m b$ (embryo-defective), are known to be required for embryo development. Their protein products often perform essential cellular functions, and any major interference with these proteins could result in embryonic lethality [3]. RUS6 is predicted to be localized in the chloroplast [24]. Normal chloroplast functions are essential to embryonic development, and studies have shown that disruptions of a number of proteins that are essential to chloroplast functions result in embryo lethality [19]. In addition, many studies have established that auxin plays a role in embryo development [25], and specific spatiotemporal distributions of auxin are well documented [25]. These distribution patterns are achieved via local auxin biosynthesis by YUCCA members or by auxin transporters (efflux or influx transporters). RUS1 and RUS2 are known to have a strong connection with auxin distributions, as both rus 1 and rus 2 mutants showed altered auxin distributions. RUS1 and RUS2 are known to play a role in PLP (pyridoxal 5-phosphate, the bioactive form of vitamin B6) homeostasis [8] and PLP homeostasis plays an important role in regulating auxin homeostasis during postembryonic root development in Arabidopsis [26]. Furthermore, previous studies suggest that RUS1 and RUS2 may physically interact with PLP-binding proteins, such as aspartate aminotransferases, to regulate vitamin B6 homeostasis. Specific mutations to the PLP-binding pocket of the ASPARTATEAMINOTRANSFERASE (ASP) proteins suppress the rus1 and rus2 phenotypes.

Our study completed the identification of T-DNA knockout mutant for all six members of the RUS gene family. No observable phenotype was detected in homozygous mutants for rus 3 , rus4, and rus5, suggesting genetic redundancy for those three $R U S$ genes. We also created double mutants for different gene pairs (rus3 rus 4 , rus 3 rus5) and no observable phenotypes were found in these double mutants (Fig. S10). Homozygous triple mutants for the three genes could be created to test if these three members indeed share functional redundancy, although the embryo lethality of rus6 presents obvious limitations with genetic crosses, and would require a transgenic line in the homozygous rus 6 background carrying RUS6 driven by an inducible promoter. Future studies on various combinations of rus3, rus4, rus5, and rus6 mutants under various physiological conditions will help determine the genetic roles of these RUS members. RUS genes are widespread in multicellular organisms and our previous phylogenetic analysis of the RUS genes suggested that RUS3 is the ortholog to the $R U S$ genes found in animals [7]. It is therefore surprising that no observable phenotypes were detected in rus3 knockout mutants in Arabidopsis under normal growth condition. Species in the animal kingdom have a single RUS gene in each genome, whereas species in the plant kingdom have multiple (5-16) RUS genes. How RUS genes function in other multicellular organisms is largely unknown. Our current study with four of the Arabidopsis $R U S$ genes provides experimental evidences that can help guide future efforts to discover how the RUS family functions in Arabidopsis and other species.

\section{Conclusion}

The Arabidopsis genome contains six RUS genes, which all encode DUF647-containing proteins. Two of these genes, RUS1 and RUS2, have roles in early seedling development, vitamin b6 homeostasis, and auxin transport. In this paper we analyzed knock-out mutants for the remaining RUS genes (RUS3 through RUS6). Interestingly, homozygous rus 3 , rus 4 , or rus 5 mutants displayed no abnormal phenotypes under standard growth conditions. However, homozygous rus6 mutant embryos failed to develop beyond the globular stage, and subsequently their seeds were aborted. RUS6 expression was detected in many phases of plant development, and was especially strong in flowers. RUS6 is an essential gene in Arabidopsis embryo development, and is likely to function throughout the plant life cycle. DUF647-containing proteins are ubiquitously present in eukaryotes and our study uncovered an essential role for one of the DUF647containing proteins in Arabidopsis embryo development.

\section{Method \\ Phylogenetic analysis}

Sequences were aligned with the MUSCLE program (MUltiple Sequence Comparison by Log-Expectation, https://www.ebi.ac.uk/Tools/msa/muscle/), and subsequently used to construct a neighbor-joining phylogenetic tree with UPGMA clustering method, distance correction and gaps excluded (https://www.ebi.ac.uk/ Tools/phylogeny/simple_phylogeny/). 
Plant materials and growth conditions

Arabidopsis seeds were surface sterilized and cold treated in darkness at $4{ }^{\circ} \mathrm{C}$ for 48 to $72 \mathrm{~h}$, then plated on Murashige and Skoog (MS) medium containing $0.5 \% \mathrm{D}$-sucrose, with a full range vitamin supplement minus B6 (except when stated otherwise). rus6/ + plants were also germinated directly in potting media with no change in phenotype. Growth conditions for Arabidopsis (ecotype Col-0) plants were as described before [7]. Seeds of T-DNA knockout lines for rus3-1 (SALK_135717C), rus4-2 (GK_447F02), rus5-1 (SALK_038772C), rus6-2 (EMB 1879) were obtained from the ABRC (Arabidopsis Biological Resource Center) at Ohio State University (https://abrc.osu.edu/). The SALK lines were donated by the Salk Institute Genomic Analysis Laboratory. The T-DNA line for rus6-1 (GK-278G06-015,156) was obtained from the University of Nottingham courtesy of Gabi-Kat (https://www.gabi-kat.de/).

\section{Seed count}

Siliques at mature stages of development $(\sim 13 \mathrm{~mm}$ in length or greater) were dissected under an Olympus SZX12 Stereozoom microscope equipped with a Qimaging micro publisher 5.0 megapixel CCD camera. Images were captured using Qcapture version 2.6 software. We noticed that the less developed siliques contained fewer brown seeds. Of those present, most were similar to the white seeds in appearance, plump with an off-white, or light brown color. In contrast, more developed siliques contained significantly more brown seeds, most of which were of a darker brown shade, and wrinkled. We suspected that a portion of the white aborted seeds took on this brown and wrinkled appearance, which increased in severity as the silique matured. To test this, we counted the number of brown wrinkled seeds present in sequential siliques along an inflorescence stem, thereby enumerating seed color and shape along a spectrum of development. Numbers of brown wrinkled seeds increase with silique development supporting that both white and brown seeds represent the same defect.

\section{Seed mounting, clearing, and observation}

Siliques at mature stages of development $(\sim 13 \mathrm{~mm}$ in length or greater) were dissected under a dissecting microscope. White and green seeds were separated and place directly onto a slide containing a drop of Hoyer's solution, which was prepared as described in [27], then diluted to one-half concentration. Siliques at immature stages of development (less than $\sim 13 \mathrm{~mm}$ in length) were dissected directly in a drop of Hoyer's solution on a slide. All seeds were incubated in solution at RT for 2-16 h until clear. Seeds were observed using a Nikon Eclipse 80i manual upright microscope with Nomarski (DIC) optics. Images were captured using Qcapture version 2.6 software.

\section{PCR genotyping}

DNA was extracted from seedlings grown on MS plates and screened for T-DNA insertions and wild-type alleles. Polymerase chain reaction was used to amplify DNA (see Supplemental Table 1 for primer list) under the following conditions: denaturation $94^{\circ} \mathrm{C}$ for $5 \mathrm{~min}$, followed by 45 cycles of $60 \mathrm{~s}$ at $95{ }^{\circ} \mathrm{C}, 30 \mathrm{~s}$ at $58{ }^{\circ} \mathrm{C}, 90 \mathrm{~s}$ at $72{ }^{\circ} \mathrm{C}$, followed by $10 \mathrm{~min}$ at $72{ }^{\circ} \mathrm{C}$. For the RUS6 GK278G06 line, primers were designed to amplify the T-DNA left border and it's genomic flanking sequence: TJLB 155 and RUS61936-F. Primers for the wild-type allele were designed to span both sides of the large (approx. 5,800 bp) T-DNA insertion, permitting amplification of the wild-type allele only: RUS6-1936-F, and RUS6-cds2-R. For the EMB 1879 line, primers were designed to amplify the T-DNA left border and it's genomic flanking sequence: GARLIC-LB3, and RUS6 RTR. Primers for the wild-type allele were designed to amplify a fragment beginning in the promoter region, and extending into exon 1: RUS6PROMOTER3-F, and RUS6-R1. Note that exon 1 is part of the proposed deletion in the EMB 1879 line, preventing RUS6-R1 from annealing to the mutant allele. 1\% $\mathrm{EtBr}$ gels were imaged using the Azure c200 gel imaging workstation.

\section{Construction of transgenic plant lines}

The promoter region of RUS6 was amplified from genomic DNA with primers RUS6-P-Kpn1-Sal1-F and RUS6 P-BamH1 (see Supplemental Table 1 for primer list) and inserted into pCR8. The RUS6 promoter insert was digested from pCR8 at Sal1 and BamH1 and inserted into a modified pBI101 with a GUS reporter completing the construct. For the GFP construct, we amplified RUS6 cDNA from an existing construct using primers AT5G49820-Kpn1-F and AT5G49820BamH1-R (see Supplemental Table 1 for primer list). This fragment was inserted into pBluescript at Kpn1 and BamH1. We subsequently inserted the RUS6 promoter insert from pCR8 at Sal1 and Kpn1. Finally, we digested the entire RUS6 promoter and cDNA insert at Kpn1 and BamH1 and ligated into a modified pZP222GFP vector.

\section{Transformation and complementation}

Agrobacterium tumefaciens (GV3101) transformed with the desired constructs was grown for $24 \mathrm{~h}$ in $50 \mathrm{~mL}$ of LB broth (with antibiotics), after inoculation 
with a $5 \mathrm{ml}$ starter. Cells were spun-down and resuspended in an equal volume of ddH20 with $0.2 \%$ Vacin-stuff (Silwet L-77). This solution was used to spray Arabidopsis flowers as the avenue of transformation. Transformed plants were incubated under clear plastic at RT in the dark for $24 \mathrm{~h}$ then returned to the growth chamber. This method was repeated one week later for a total of two transformation events. rus6/ + GK278G06 plants were complemented by the chimeric AT5G498920 (RUS6)-GFP gene driven by a RUS6 promoter in the construct pZP222-GFP. Seeds were harvested and plated on Sul+, Gen + MS media for antibiotic selection. Two T-1 Transformants and multiple T-2 (complemented) progeny were recovered and confirmed by PCR analysis. WT plants were transformed with the modified pBI101 GUS reporter construct driven by a RUS6 promoter using the same methods above. T-1 seeds were harvested and plated on Kan + MS media for antibiotic selection. Twelve T-1 Transformants and multiple T-2 progeny were recovered and confirmed by PCR analysis.

\section{GUS staining}

All plant tissues were vacuum infiltrated for thirty minutes with GUS staining solution, (1 mg 5-bromo-4chloro-3-indolyl $\beta$-D-Glucuronide (X-Gluc) dissolved in $0.1 \mathrm{~mL}$ methanol, $1 \mathrm{~mL} 2 \times$ buffer $(20 \mu \mathrm{L} 0.1 \mathrm{M}$ potassium ferrocyanide, $20 \mu \mathrm{L} 0.1 \mathrm{M}$ potassium ferricyanide), $10 \mu \mathrm{L}$ $10 \%(\mathrm{w} / \mathrm{v})$ solution of Triton $\mathrm{X}-100,0.85 \mathrm{~mL}$ water), then incubated overnight at $37^{\circ} \mathrm{C}$. Samples were cleared using $70 \%$ ethanol. All samples were observed using an Olympus SZX12 Stereozoom microscope equipped with a Qimaging micro publisher 5.0 megapixel CCD camera. Images were captured using Qcapture version 2.6 software.

\section{RUS6 subcellular location RUS6-GFP}

RUS6-GFP complemented and wild-type control seeds were dissected and mounted in a $6 \%$ glycerol solution, embryos were extruded by tapping the slide as described by [27]. Embryos were observed using a Zeiss LSM 710 Confocal Laser Scanning Microscope. GFP fluorescence was excited by a blue argon laser (488-nm blue excitation) and detected at 515- to 530$\mathrm{nm}$ wavelengths. Images were processed using Fiji ImageJ [28].

\footnotetext{
Abbreviations

DUF: Domain of unknown function; EMB: Embryo-defective; GFP: Green fluorescence protein; GUS: $\beta$-Glucuronidase; PLP: Pyridoxal 5-phosphate; MS medium: Murashige and Skoog medium; RUS: Root UV-B sensitive; Sul: Sulfadiazine; WXR: Weak Auxin response; X-Gluc: 5-Bromo-4-chloro-3-indolyl $\beta$-D-Glucuronide.
}

\section{Supplementary Information}

The online version contains supplementary material available at https://doi. org/10.1186/s12870-021-03011-8.

Additional file 1.

\section{Acknowledgements}

We would like to thank Dr. David Meinke of the SeedGenes (seedgenes.org) for providing information about the emb1879 line. We would like to thank Dr. Annette Chan of the Cell and Molecular Imaging Center (CMIC) at the College of Science and Engineering of San Francisco State University for helping us with Confocal imaging. Thanks also go to other He Lab members of the past and the present who provided valuable insights to this study; Cinthya Ibarra, Nathan O'Neil, Keirstinne Turcios, Dylan Ting, Stacey Phan, Claudia Bucheli, Brendan Heath, Susan Prestol, and Anna Calamonaci.

\section{Authors' contributions}

N. P.; H.T. and C. L. performed the experiments with the assistance of E. M. D. N. P.; C. L. and Z.-H. H. conceived the project and Z.-H. H. supervised the project; Z.-H. H. secured the funding. N. P.; Z.-H. H. and C. L. wrote the article with contributions of all the authors. All authors have read and approved the manuscript.

\section{Funding}

This study was supported by a Bridges Funding from the Office of Research and Sponsored Programs of San Francisco State University. The funding organization provided the financial support to the research projects, but was not involved in the design of the study, data collection, analysis of the data, or the writing of the manuscript.

\section{Availability of data and materials}

Linked genotype and phenotype data used and/or analyzed during the current study are deposited at TAIR (The Arabidopsis Information Resourcearabidopsis.org). The linked genotype and phenotype for the four genes (RUS3, RUS4, RUS5, RUS6) can be found at these links:

RUS3 https://www.arabidopsis.org/servlets/TairObject?id=28854\&type=locus RUS4 https://www.arabidopsis.org/servlets/TairObject?id=32619\&type=locus RUS5 https://www.arabidopsis.org/servlets/TairObject?id=131341\&type $=$ locus

RUS6 https://www.arabidopsis.org/servlets/TairObject?id=132107\&type= locus

\section{Declarations}

Ethics approval and consent to participate

Not applicable.

\section{Consent for publication}

Not applicable.

\section{Competing interests}

The authors declare that they have no competing interests.

Received: 30 September 2020 Accepted: 5 May 2021

Published online: 25 May 2021

\section{References}

1. Castle LA, Errampalli D, Atherton TL, Franzmann LH, Yoon ES, Meinke DW. Genetic and molecular characterization of embryonic mutants identified following seed transformation in Arabidopsis. Molec Gen Genet. 1993;241-241:504-14. https://doi.org/10.1007/BF00279892.

2. Tzafrir I, Pena-Muralla R, Dickerman A, Berg M, Rogers R, Hutchens S, et al. Identification of genes required for embryo development in Arabidopsis. Plant Physiol. 2004;135:1206-20. https://doi.org/10.1104/pp.104. 045179. 
3. Meinke DW. Genome-wide identification of EMBRYO-DEFECTIVE (EMB) genes required for growth and development in Arabidopsis. New Phytol. 2020;226:306-25. https://doi.org/10.1111/nph.16071.

4. Franzmann L, Patton DA, Meinke DW. In vitro morphogenesis of arrested embryos from lethal mutants of Arabidopsis thaliana. Theoret Appl Genetics. 1989;77:609-16. https://doi.org/10.1007/BF00261231.

5. McElver J, Tzafrir I, Aux G, Rogers R, Ashby C, Smith K, et al. Insertional mutagenesis of genes required for seed development in Arabidopsis thaliana. Genetics. 2001;159:1751-63.

6. Tong H, Leasure CD, Hou X, Yuen G, Briggs W, He Z-H. Role of root UV-B sensing in Arabidopsis early seedling development. Proc Natl Acad Sci USA. 2008;105:21039-44. https://doi.org/10.1073/pnas.0809942106.

7. Leasure CD, Tong H, Yuen G, Hou X, Sun X, He Z-H. ROOT UV-B SENSITIVE2 acts with ROOT UV-B SENSITIVE1 in a root ultraviolet B-sensing pathway. Plant Physiol. 2009;150:1902-15. https://doi.org/10.1104/pp.109.139253.

8. Leasure CD, Tong H-Y, Hou X-W, Shelton A, Minton M, Esquerra R, et al. root uv-b sensitive mutants are suppressed by specific mutations in ASPARTATE AMINOTRANSFERASE2 and by exogenous vitamin B6. Mol Plant. 2011;4:759-70. https://doi.org/10.1093/mp/ssr033.

9. Ge L, Peer W, Robert S, Swarup R, Ye S, Prigge M, et al. Arabidopsis ROOT UVB SENSITIVE2/WEAK AUXIN RESPONSE1 is required for polar auxin transport. Plant Cell. 2010;22:1749-61. https://doi.org/10.1105/tpc.110. 074195.

10. Yu H, Karampelias M, Robert S, Peer WA, Swarup R, Ye S, et al. ROOT ULTRAVIOLET B-SENSITIVE1/weak auxin response3 is essential for polar auxin transport in Arabidopsis. Plant Physiol. 2013;162:965-76. https:// doi.org/10.1104/pp.113.217018.

11. Zhang X, Carter MS, Vetting MW, San Francisco B, Zhao S, Al-Obaidi NF, et al. Assignment of function to a domain of unknown function: DUF1537 is a new kinase family in catabolic pathways for acid sugars. Proc Natl Acad Sci USA. 2016;113:E4161-9. https://doi.org/10.1073/pnas.16055 46113.

12. Goodacre NF, Gerloff DL, Uetz P. Protein domains of unknown function are essential in bacteria. mBio. 2014;5:e00744-13. https://doi.org/10.1128/ mBio.00744-13.

13. Jiao C, Sørensen I, Sun X, Sun H, Behar H, Alseekh S, et al. The penium margaritaceum genome: hallmarks of the origins of land plants. Cell. 2020;181(1097-1111):e12. https://doi.org/10.1016/j.cell.2020.04.019.

14. Kleinboelting N, Huep G, Kloetgen A, Viehoever P, Weisshaar B. GABI-Kat SimpleSearch: new features of the Arabidopsis thaliana T-DNA mutant database. Nucleic Acids Res. 2012;40:D1211-5. https://doi.org/10.1093/ nar/gkr1047.

15. Meinke DW. Embryo-lethal mutants of Arabidopsis thaliana: analysis of mutants with a wide range of lethal phases. Theor Appl Genet. 1985;69-69:543-52. https://doi.org/10.1007/BF00251102.

16. Emanuelsson $\mathrm{O}$, Nielsen $\mathrm{H}$, Brunak S, von Heijne G. Predicting subcellular localization of proteins based on their $\mathrm{N}$-terminal amino acid sequence. J Mol Biol. 2000;300:1005-16. https://doi.org/10.1006/jmbi.2000.3903.
17. Smyth DR, Bowman JL, Meyerowitz EM. Early flower development in Arabidopsis. Plant Cell. 1990;2:755-67. https://doi.org/10.1105/tpc.2.8. 755.

18. Zhao S-Q, Li W-C, Zhang Y, Tidy AC, Wilson ZA. Knockdown of Arabidopsis ROOT UVB SENSITIVE4 disrupts anther dehiscence by suppressing secondary thickening in the endothecium. Plant Cell Physiol. 2019;60:2293306. https://doi.org/10.1093/pcp/pcz127.

19. Bryant N, Lloyd J, Sweeney C, Myouga F, Meinke D. Identification of nuclear genes encoding chloroplast-localized proteins required for embryo development in Arabidopsis. Plant Physiol. 2011;155:1678-89. https://doi.org/10.1104/pp.110.168120.

20. Tambasco-Studart M, Titiz O, Raschle T, Forster G, Amrhein N, Fitzpatrick TB. Vitamin B6 biosynthesis in higher plants. Proc Natl Acad Sci U S A. 2005;102:13687. https://doi.org/10.1073/pnas.0506228102.

21. Le BH, Cheng C, Bui AQ, Wagmaister JA, Henry KF, Pelletier J, et al. Global analysis of gene activity during Arabidopsis seed development and identification of seed-specific transcription factors. Proc Natl Acad Sci. 2010;107:8063-70. https://doi.org/10.1073/pnas.1003530107.

22. Swanson R, ClarkT, Preuss D. Expression profiling of Arabidopsis stigma tissue identifies stigma-specific genes. Sex Plant Reprod. 2005;18:163-71. https://doi.org/10.1007/s00497-005-0009-X.

23. Narsai R, Law SR, Carrie C, Xu L, Whelan J. In-depth temporal transcriptome profiling reveals a crucial developmental switch with roles for RNA processing and organelle metabolism that are essential for germination in Arabidopsis. Plant Physiol. 2011;157:1342-62. https://doi.org/10.1104/ pp.111.183129.

24. Kaundal R, Saini R, Zhao PX. Combining machine learning and homologybased approaches to accurately predict subcellular localization in Arabidopsis. Plant Physiol. 2010;154:36-54. https://doi.org/10.1104/pp. 110.156851.

25. Figueiredo DD, Köhler C. Auxin: a molecular trigger of seed development Genes Dev. 2018;32:479-90. https://doi.org/10.1101/gad.312546.118.

26. Boycheva S, Dominguez A, Rolcik J, Boller T, Fitzpatrick TB. Consequences of a deficit in vitamin $B 6$ biosynthesis de novo for hormone homeostasis and root development in Arabidopsis. Plant Physiol. 2015;167:102-17. https://doi.org/10.1104/pp.114.247767.

27. Feng J, Ma L. A method for characterizing embryogenesis in Arabidopsis. J Vis Exp. 2017. https://doi.org/10.3791/55969.

28. Schindelin J, Arganda-Carreras I, Frise E, Kaynig V, Longair M, Pietzsch T, et al. Fiji: an open-source platform for biological-image analysis. Nat Methods. 2012;9:676-82. https://doi.org/10.1038/nmeth.2019.

\section{Publisher's Note}

Springer Nature remains neutral with regard to jurisdictional claims in published maps and institutional affiliations.
Ready to submit your research? Choose BMC and benefit from:

- fast, convenient online submission

- thorough peer review by experienced researchers in your field

- rapid publication on acceptance

- support for research data, including large and complex data types

- gold Open Access which fosters wider collaboration and increased citations

- maximum visibility for your research: over 100M website views per year

At BMC, research is always in progress.

Learn more biomedcentral.com/submissions 